\title{
Fracture Surface Analysis of 3D-Printed Tensile Specimens of Novel ABS-Based Materials
}

\author{
Angel R. Torrado Perez • David A. Roberson • \\ Ryan B. Wicker
}

Submitted: 30 October 2013/in revised form: 27 January 2014/Published online: 20 March 2014

(C) ASM International 2014

\begin{abstract}
One of the most common materials utilized by material extrusion 3D printing is acrylonitrile butadiene styrene (ABS). The work presented in this research explored the effect of the addition of reinforcing materials on the mechanical properties of ABS in an effort to create materials with enhanced physical properties. A comparison was made between pure ABS, two ABS matrix composites, and one ABS/elastomer blend with the purpose of characterizing the effect of additives on the mechanical properties. Tensile test results of specimens built in different orientations showed that ABS reinforced with $5 \%$ by weight $\mathrm{TiO}_{2}$ exhibited the highest ultimate tensile strength for specimens built in both horizontal and vertical directions with 32.2 and $18.4 \mathrm{MPa}$, respectively. The compounding of an elastomeric material with ABS improved the surface finish of parts as they were visibly smoother compared to those printed from the ABS baseline material, though there was an observable decrease in the ductility of tensile specimens. Analysis was performed on the fracture surface of the tensile specimens through the use of scanning electron microscopy. Fractography revealed different modes of failure related to the different additives. The effects of additives on the anisotropy associated with the mechanical properties of 3D-printed parts were also analyzed.
\end{abstract}

Keywords 3D printing - Additive manufacturing . Composite $\cdot$ Polymer blend $\cdot$ Fused deposition modeling . Fractography · Fracture

A. R. Torrado Perez $(\square)$ - D. A. Roberson - R. B. Wicker Material Science, University of Texas at El Paso, $500 \mathrm{~W}$ University Ave 5525 N. Stanton St. Apt 26D, El Paso, TX 79902, USA

e-mail: artorradoperez@miners.utep.edu; artorrado@gmail.com

\section{Introduction}

Additive manufacturing, now more commonly referred to as $3 \mathrm{D}$ printing (3DP), has gained acceptance and popularity in manufacturing, educational, and home-use settings [1,2]. Material extrusion 3D printers similar in function to the trademarked fused deposition modeling (FDM) process are the most common type of equipment used in 3DP and rely on a process by which a polymeric filament is extruded and deposited in a layer-by-layer manner until a 3D object is created. Parts are fabricated from a thermoplastic polymer that has rubbery, tacky phase above the glass transition temperature and facilitates fusion between subsequent layers. Currently, the number of polymers compatible with material extrusion 3DP platforms is very limited due to the particular properties needed for a successful print such as a relatively low glass transition temperature $\left(T_{\mathrm{g}}\right)$, melting point $\left(T_{\mathrm{m}}\right)$, and a low tendency to shrink upon solidification. The $T_{\mathrm{g}}$ will have an effect on how easily the material will be extruded, how the parts will shrink during the cooling process (therefore, affecting the warping, but not being the only one) and the thermostability of the final part. The $T_{\mathrm{m}}$ can provide some clues about the extrusion temperature $\left(T_{\mathrm{e}}\right)$, but the final $T_{\mathrm{e}}$ will depend greatly on the configuration of the feeding system in the machine. Most of the 3D printers nowadays are able to work at temperatures of less than $300^{\circ} \mathrm{C}$. Two of the most widely used materials for material extrusion 3DP are acrylonitrile butadiene styrene (ABS, $T_{\mathrm{g}}=110{ }^{\circ} \mathrm{C}$, not a true melting point) and polylactic acid (PLA, $T_{\mathrm{g}}=60^{\circ} \mathrm{C}, T_{\mathrm{m}}=175^{\circ} \mathrm{C}$ ) because of their dimensional stability and low $T_{\mathrm{g}}$. Other printable polymers are polycarbonate $\left(\mathrm{PC}, T_{\mathrm{g}}=145^{\circ} \mathrm{C}, T_{\mathrm{m}}=230-260{ }^{\circ} \mathrm{C}\right)$, polyvinyl alcohol (PVA, $T_{\mathrm{g}}=85^{\circ} \mathrm{C}, T_{\mathrm{m}}=170{ }^{\circ} \mathrm{C}$ ), and polythermide (Ultem, $T_{\mathrm{g}}=185-216{ }^{\circ} \mathrm{C}, \quad T_{\mathrm{m}}=$ $350-400{ }^{\circ} \mathrm{C}$ ), but the use of such materials presents some 
limitations. These materials require higher extrusion temperatures, over $300{ }^{\circ} \mathrm{C}$. Other specific requirements in order to decrease warpage (due to the higher gradients of temperature to be cooled) are temperature-controlled build envelopes, preheated platforms and vacuum platforms, or the use of adhesive materials.

Since the number of usable polymer types is limited, the number of applications that can benefit from material extrusion 3DP is as well limited. A strategy for increasing the applicability of material extrusion 3DP is the development of new material systems with a wider range of physical properties. A logical path to engineering the physical properties of materials used in 3DP is the development of composites where the matrix material is a printable polymer to maintain compatibility with material extrusion 3D printers. There are multiple examples of successful implementation of such material modifications for use in $\mathrm{FDM}^{\mathrm{TM}}$ [3-7].

The development of polymer matrix composites (PMC) is an obvious path to developing better materials for use in material extrusion 3D printers due to the ease of material blending and compounding, combined with the generally low prices of the matrix materials (less than a dollar per pound for ABS). Moreover, the ability to make a composite monofilament compatible with material extrusion 3D printers through conventional screw extrusion equipment offers rapid data turns in novel materials development. The augmenting of polymeric materials can be done in many ways, one being the compounding of particulate or fiberreinforcing additives in the creation of PMC, or through the compounding with other polymeric materials in the creation of polymer blends. Particles give the flexibility to tailor the characteristics of the resulting composite to fill a variety of applications based on many different factors. Parameters such as the particle size, the particle loading percentage, or the interfacial adhesion can affect the mechanical properties such as the ultimate tensile strength (UTS), the Young's modulus or the fracture toughness, and other properties including the thermostability, the coefficient of thermal expansion, decomposition temperature, or even flame-retardant characteristics $[8,9]$.

As new composite material systems are developed for use in material extrusion 3D printers, understanding the effect of additives on the mechanical behavior of the polymeric matrix is paramount. An important aspect of composite materials development is the characterization of the influence of reinforcing agents on the mechanical properties (in comparison with the material alone) and their relation with the fracture morphology of the failed components, namely, the characterization of the effect of reinforcing agents on the mechanical properties (compared with the matrix material alone) and a correlation of changes to the fracture morphology of the failed components
Table 1 Parameters of extrusion for ABS composite filaments

\begin{tabular}{ll}
\hline Temperature zone $1,{ }^{\circ} \mathrm{C}$ & 160 \\
Temperature zone $2,{ }^{\circ} \mathrm{C}$ & 205 \\
Temperature zone $3,{ }^{\circ} \mathrm{C}$ & 225 \\
Temperature zone $4,{ }^{\circ} \mathrm{C}$ & 230 \\
Temperature zone $5,{ }^{\circ} \mathrm{C}$ & 230 \\
Speed on main screws, rpm & 35 \\
Speed on feeding screws, $\%$ & 8 \\
Pressure on main screws, bar & 25 \\
Load, $\%$ & 63 \\
\hline
\end{tabular}

are important aspects to consider. The objective of the work performed in this paper is to explore the effect of additives on tensile testing data and fracture surface morphology for two ABS matrix-printable composites and one ABS/elastomer blend subjected to tensile testing. The effect of build orientation on the mechanical properties and fracture surface was also analyzed, as one of the major flaws of additive manufacturing is the anisotropy on the 3D-printed items. Three different additives were chosen for this investigation: (1) $\mathrm{TiO}_{2}$ for a particle-loaded composite; (2) jute fiber for short fiber reinforcement based on its green manufacturing composition [10]; and (3) a thermoplastic elastomer (TPE) to explore the rubber-toughening effect when blended with ABS [11].

\section{Experimental Procedure}

Monofilaments were produced using a Dr. Collin Twin Screw Extruder/Compounder Model ZK 25T (Dr. Collin $\mathrm{GmbH}$, Ebersberg, Germany) with co-rotating, intermeshing screws. Two composite types were produced utilizing $\mathrm{ABS}$ as a matrix material: ABS $\left(\mathrm{Cyclolac}^{\circledR}, \mathrm{GE}\right.$ ABS resin) loaded with 5 wt.\% jute fiber, and ABS loaded with $5 \mathrm{wt} . \% \mathrm{TiO}_{2}$. In addition, a polymeric blend was obtained by mixing ABS with 5 wt.\% of a TPE. The filament was produced to be compatible with the MakerBot Replicator (MakerBot Industries, Brooklyn, NY USA) material extrusion 3D Printer and possessed a diameter of $1.77 \mathrm{~mm}$. The three ABS-based materials were compared with the ABS filament provided by MakerBot Industries. The compounded materials were produced with the same extrusion parameters, as represented in Table 1. Micrographs were taken of the additives before the processing using a Hitachi TM-1000 scanning electron microscope (SEM) (Hitachi High-Technologies Europe GmbH, Germany) operating at $15 \mathrm{kV}$. Images are shown in Fig. 4.

The specimens were printed following the Type $\mathrm{V}$ dimensions described by the American Society for Testing and Materials (ASTM) D638 standard [12] and verified to fulfill the tolerance requirements. All specimens were 
Fig. 1 ASTM 638, type V: dimensions

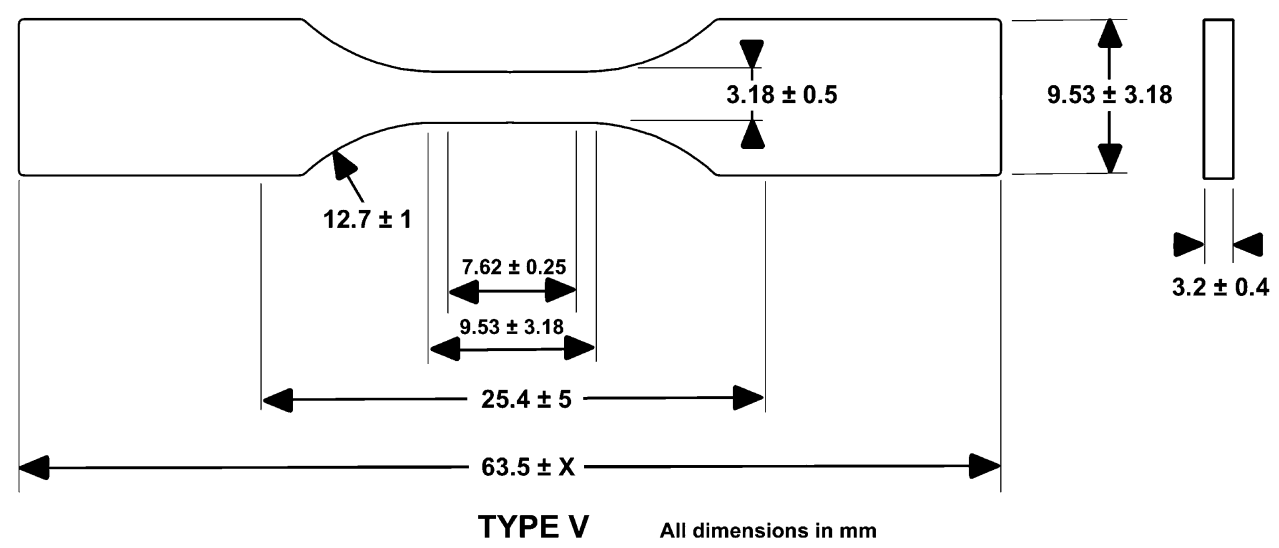

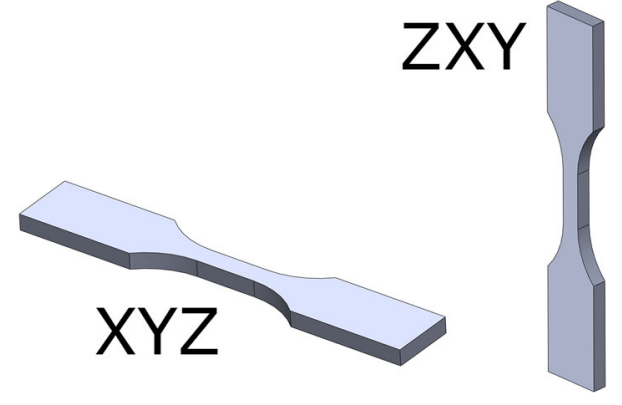

Fig. 2 Printing directions

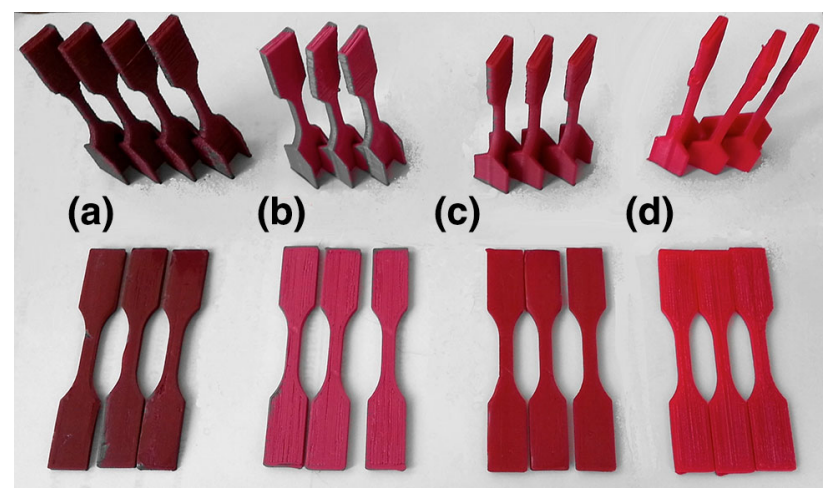

Fig. 3 Samples (vertical above, horizontal below): (a) ABS and jute fiber, (b) $\mathrm{ABS}$ and $\mathrm{TiO}_{2}$, (c) ABS with TPE, and (d) pure ABS

created in the same area on the machine's platform to minimize variability due to possible temperature gradients inside the build envelope. The fabrication orientation on the machine as well as the print raster path (also known as fill pattern) can play an important role on the mechanical properties of the fabricated part due to the anisotropic nature of this fabrication technique [13-15]. For this reason, two sets of specimens were produced for each material type, one printed in the XYZ direction and another set printed in the ZXY direction as seen in Figs. 1 and 2. The testing samples produced from the four material types were
Table 2 Extrusion parameters used with MakerBot replicator

\begin{tabular}{ll}
\hline Object infill, $\%$ & 100 \\
\hline Layer height, mm & 0.27 \\
Number of shells & 1 \\
Feedrate, $\mathrm{mm} / \mathrm{s}$ & 40 \\
Travel feedrate, $\mathrm{mm} / \mathrm{s}$ & 55 \\
Print temperature, ${ }^{\circ} \mathrm{C}$ & 230 \\
Filament diameter, $\mathrm{mm}$ & 1.9 \\
Nozzle diameter, $\mathrm{mm}$ & 0.4 \\
Raft material & No \\
\hline
\end{tabular}

fabricated with the same parameters on the machine, which were previously iterated to get the optimum filling on the specimen without leading to dragging of the part due to an excess of material deposited to obtain, a part with the lower amount of air gaps in between the deposited threads (Fig. 3). Table 2 shows the values for the parameters utilized in the 3D printer. Figure 4 shows the SEM micrographs of the raw additives before the processing.

An Instron ${ }^{\circledR} 5866$ (Instron, Norwood, MA, USA) tensile testing machine equipped with a $10 \mathrm{kN}$ load cell was utilized to perform the tensile testing. The specimens were tested at a speed of $10 \mathrm{~mm} / \mathrm{min}$ and a temperature of $23{ }^{\circ} \mathrm{C}$. An Instron ${ }^{\circledR}$ 2663-821 advanced video extensometer (AVE) was used to determine the instant strain at every moment, allowing for the plotting of the entire stress-strain curve and the automatic calculation of the modulus and the $\%$ elongation to break. The distance between the marks of 7.6 was used for the AVE. Figures 5 and 6 show the stressstrain curves results for the four material types produced. The stress-strain curves were plotted based on the average results from a sample size of five specimens. The data extracted from the AVE and the load cell were processed with a program developed in Matlab ${ }^{\circledR}$ which homogenized the data in order to generate stress-strain curves. The program allowed for the plotting of a single stress-strain 

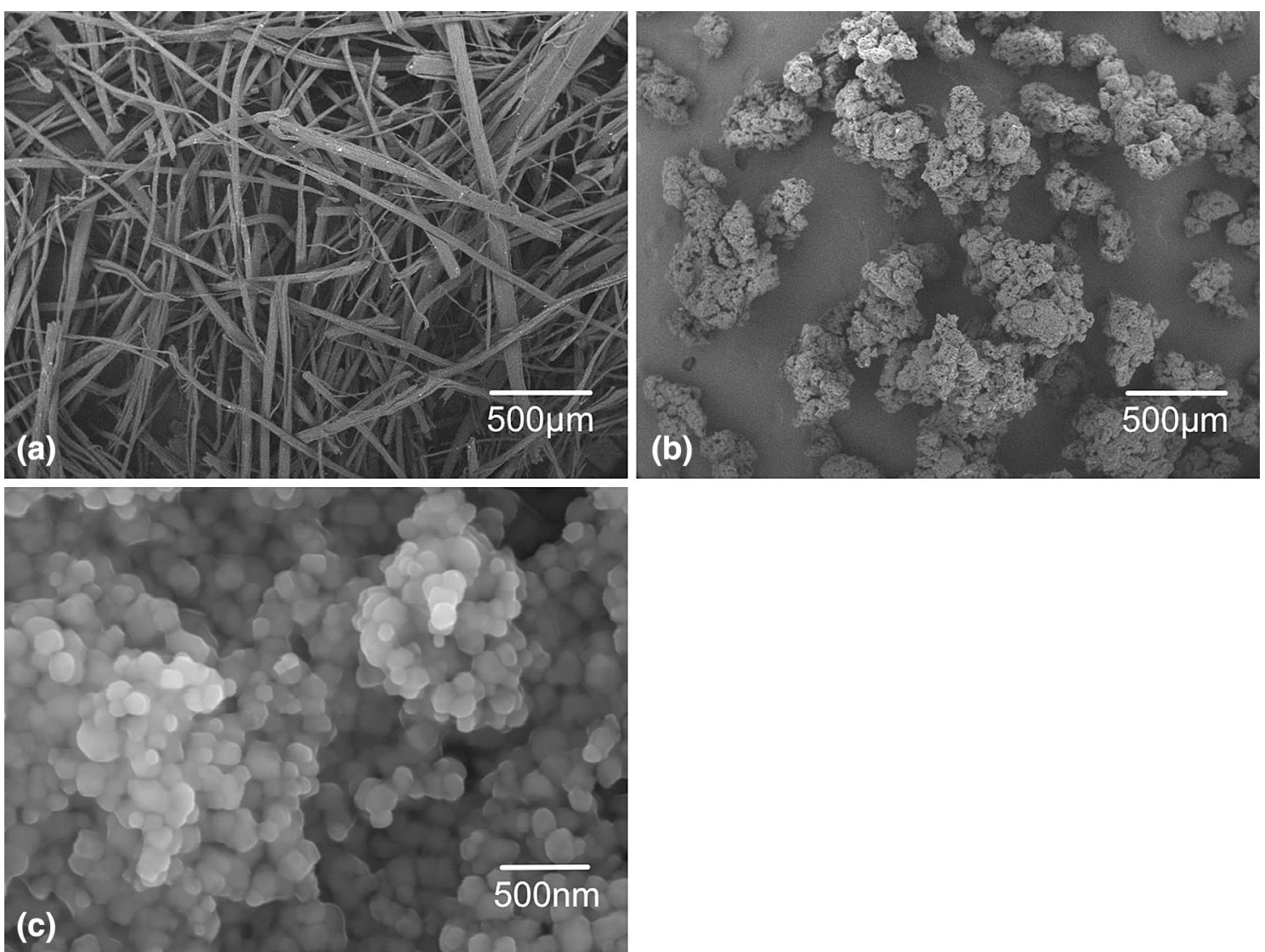

Fig. 4 Additives: (a) jute fibers, (b) TPE, and (c) $\mathrm{TiO}_{2}$
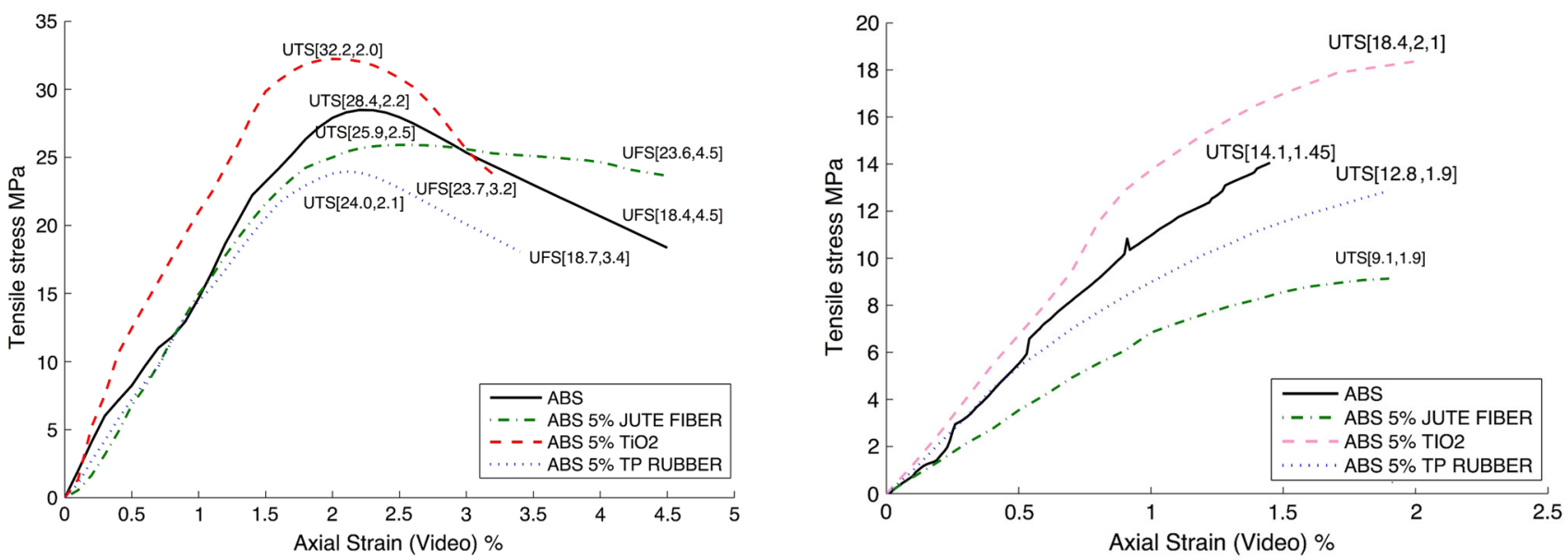

Fig. 5 Stress-strain curves: XYZ direction

Fig. 6 Stress-strain curves: ZXY direction

\section{Tensile Test Results}

curve for each sample set by calculating a composite of the stress-strain curves for all the specimens in a given sample pool. Figure 7 shows an example of the resulting curve for the ABS specimen sample set tested in this study. The fracture surfaces were analyzed via SEM.

\section{$\mathrm{ABS} / \mathrm{TiO}_{2}$ Composite}

The composite prepared from ABS in combination with $\mathrm{TiO}_{2}$ was the only sample pool which demonstrated an 


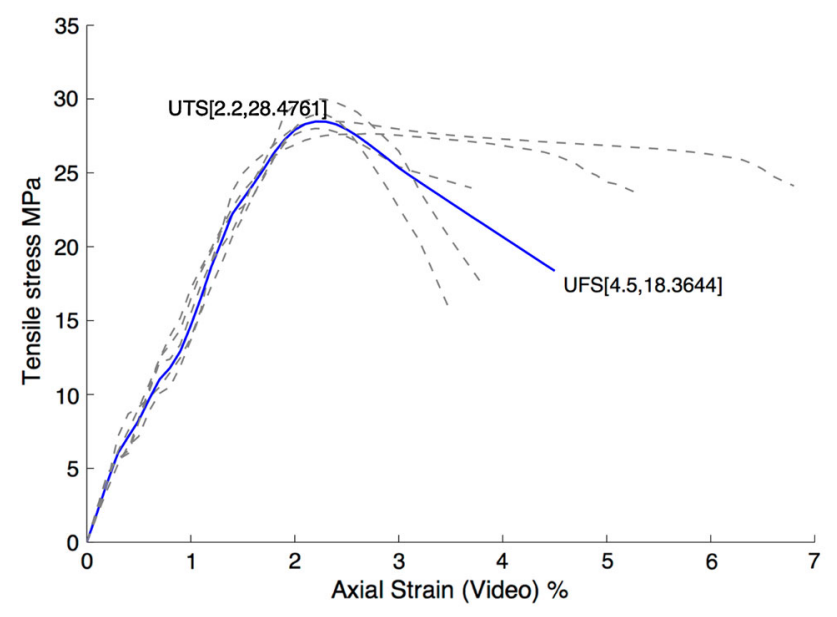

Fig. 7 Stress-strain curve averaging with Matlab $^{\circledR}$

improvement in UTS with an increase of $13.2 \%$ in comparison with the commercially available ABS. Moreover, the strength at fracture was on average $30 \%$ higher, while the strain at fracture was reduced by $29 \%$. The UTS in the ZXY direction was improved by $30 \%$, while the strain was increased by $45 \%$ compared with samples made from ABS filament. The parts produced from $\mathrm{ABS} / \mathrm{TiO}_{2}$ also exhibited a lower roughness than samples fabricated from pure ABS.

\section{ABS/TPE Blend}

The blending of ABS with TPE had the effect of reducing the UTS by $16 \%$ in the case of parts printed in XYZ direction and also reduced the UTS by $9 \%$ in the case of parts printed in the ZXY direction compared with baseline ABS samples. The percent elongation for ABS/TPE samples did not deviate compared with ABS for components printed in the $\mathrm{XYZ}$ direction, however parts printed in the ZXY direction were able to withstand $31 \%$ more strain compared to those printed from pure ABS. The stressstrain curve indicated the modulus of the ABS/TPE blend was the same as the modulus observed for tensile testing of ABS printed in the XYZ direction, not modifying the behavior in the linear-elastic region; however, the UTS was reduced. Even though the mechanical properties were compromised, blending ABS with TPE resulted in an improvement on the final surface finish and a reduction in warping during the printing process.

\section{ABS/Jute Fiber Composite}

The compounding of jute fiber with ABS had the effect of reducing the UTS by $9 \%$ but improved the amount of plastic deformation increasing the strength at fracture by $28 \%$ in the case of samples printed in the XYZ direction.
Table 3 Values of UTS and UFS for XYZ specimens

\begin{tabular}{lccccc}
\hline & & & \multicolumn{3}{c}{$\begin{array}{c}\text { Young's modulus, } \\
\text { MPa }\end{array}$} \\
\cline { 5 - 7 } Material & \begin{tabular}{c} 
Utress, MPa \\
\cline { 5 - 6 }
\end{tabular} & $\begin{array}{c}\text { Stress, MPa } \\
\text { S Strain, \% }\end{array}$ & @ Strain, \% & Average & St. Dev. \\
\hline ABS & $28.5 @ 2.2$ & $18.4 @ 4.5$ & 1530 & 114 \\
ABS 5\% jute & $25.9 @ 2.5$ & $23.6 @ 4.5$ & 1543 & 121 \\
ABS 5\% $\mathrm{TiO}_{2}$ & $32.2 @ 2.0$ & $23.8 @ 3.2$ & 1708 & 121 \\
ABS 5\% TP rubber & $24.0 @ 2.1$ & $18.1 @ 3.4$ & 1580 & 113 \\
\hline
\end{tabular}

Table 4 Values of UTS and UFS for vertical specimens

\begin{tabular}{llccc}
\hline \multirow{2}{*}{ Material } & UTS/ UFS & & \multicolumn{2}{c}{ Young's modulus, MPa } \\
\cline { 4 - 5 } & Stres, MPa & Strain (\%) & Average & St. Dev. \\
\hline ABS & $14.1 @ 1.5$ & 1190 & 166 \\
ABS 5\% jute & $12.9 @ 1.9$ & 871 & 234 \\
ABS 5\% TiO & $18.4 @ 2.0$ & 1355 & 244 \\
ABS 5\% TP rubber & $9.1 @ 1.9$ & 1101 & 300 \\
\hline
\end{tabular}

For samples printed in the ZXY direction, the addition of jute fiber reduced the fracture strength by $35 \%$ and increased the fracture strain by $31 \%$. Parts fabricated from the jute composite exhibited the highest roughness of the four compared materials. It was also observed that the addition of jute to ABS decreased the amount of warping, leading to greater dimensional stability.

The results of tensile testing are represented in Tables 3 and 4. Overall, parts fabricated in the ZXY direction were able to withstand less plastic deformation than those fabricated in the XYZ direction. There is a precedent in the literature for the characterization of the effect of mechanical properties on build orientation for components fabricated from material extrusion 3DP [13-15].

\section{Fractography}

The breakage occurred in the gage section for the all the samples tested. Figure 8 shows a representative tested sample from each sample set. The fracture surfaces were analyzed for samples printed in both the horizontal XYZ and vertical ZXY directions in low magnification (Figs. 9, 11) and high magnification (Figs. 10, 12). Representative electron micrographs of the fracture surfaces for the four material systems studied in this paper demonstrated drastically different fracture behaviors. In most cases, the fracture of a thermoplastic component is ductile due to the reorientation and stretching of the thread-like macromolecules that allow for high deformation on the material. In contrast, the fracture surfaces of thermoset polymeric components 
typically do not exhibit a high amount of plastic deformation as their macromolecules possess a relatively high level cross-linkage between the polymeric chains. Elastomers tend to fracture after a high amount of elastic distortion, leaving a very slight residual deformation [16]. In our case, we were dealing with the examination of thermoplastics and a thermoplastic/elastomer blend, and so one would expect to see fracture characteristics typical of ductile fracture.

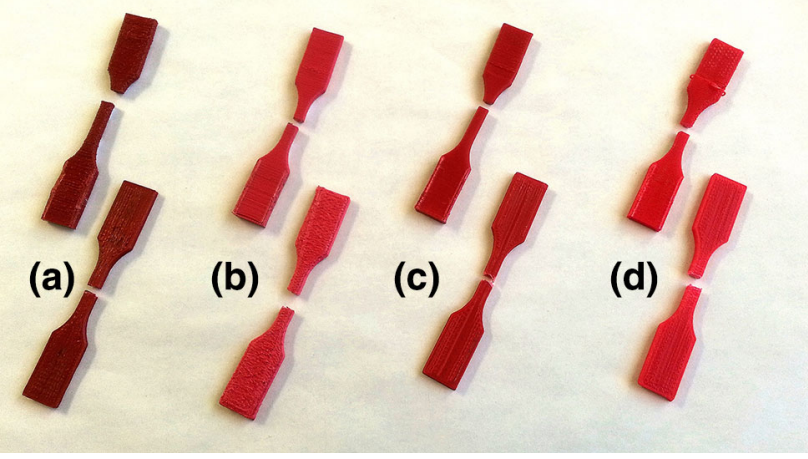

Fig. 8 Broken specimens after tensile test (vertical above, horizontal below): (a) $\mathrm{ABS}$ and jute fiber, (b) $\mathrm{ABS}$ and $\mathrm{TiO}_{2}$, and (c) $\mathrm{ABS}$ with TPE, (d) pure ABS
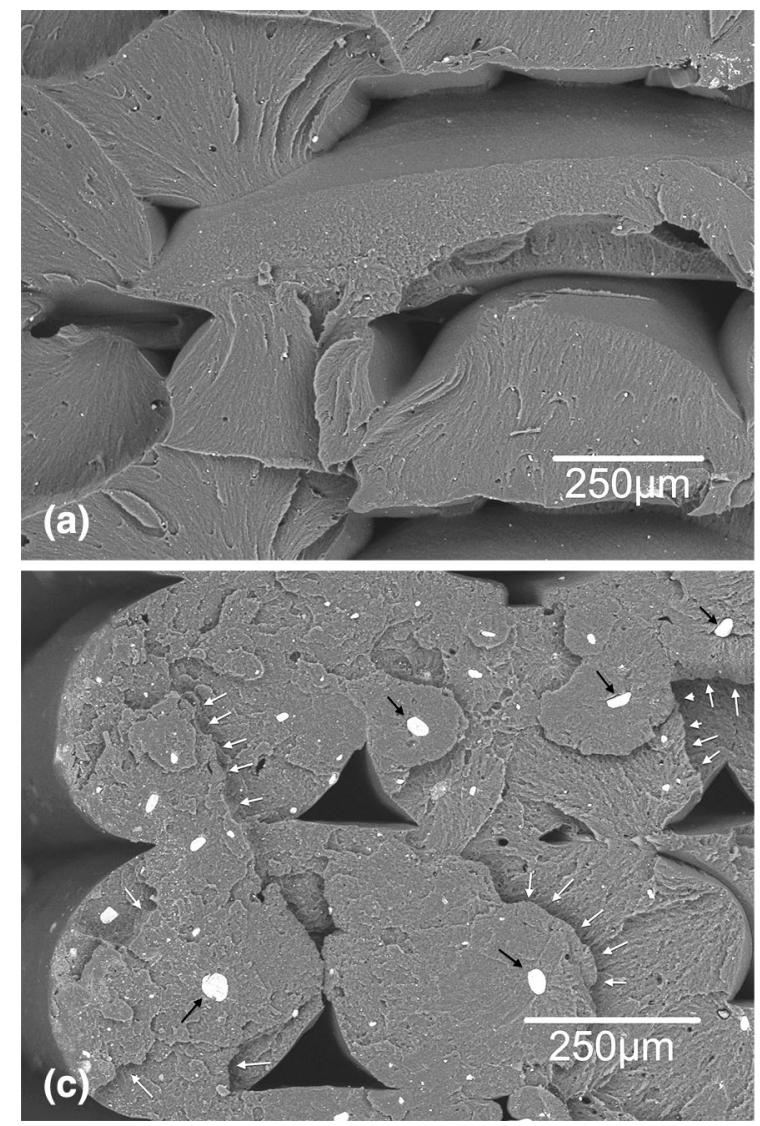

\section{Fractography of Samples Printed in the XYZ Direction}

Voids are commonly found in material extrusion 3DP between the deposited print rasters. However, a different level of filling was achieved depending on the unique characteristics of the molten composite.

The fracture surface of the baseline sample fabricated from ABS is characteristic of ductile fracture observed in thermoplastic materials (Figs. 9a, 10a). As can be seen in the micrographs, several opened-up crazes were generated by tear fractures during the deformation of the continuous threads. Figure 10a shows a V-shaped ramp characteristic of a tear fracture that is typically generated on the surface and then propagates inward [16].

Multiple craters and voids can be observed on the surface fracture of ABS loaded with jute fiber (Figs. 9b, 10b). Jute fiber has been reported to undergo decomposition starting at temperatures of $180{ }^{\circ} \mathrm{C}$ [17]. The breakdown of the cellulose would lead to secondary byproducts within the mixture and the generation of combustion gases that would remain trapped within the filament during the extrusion process, depending on the time of exposure to the high temperatures. Even if the decomposition process was not completed during the compound of the monofilament
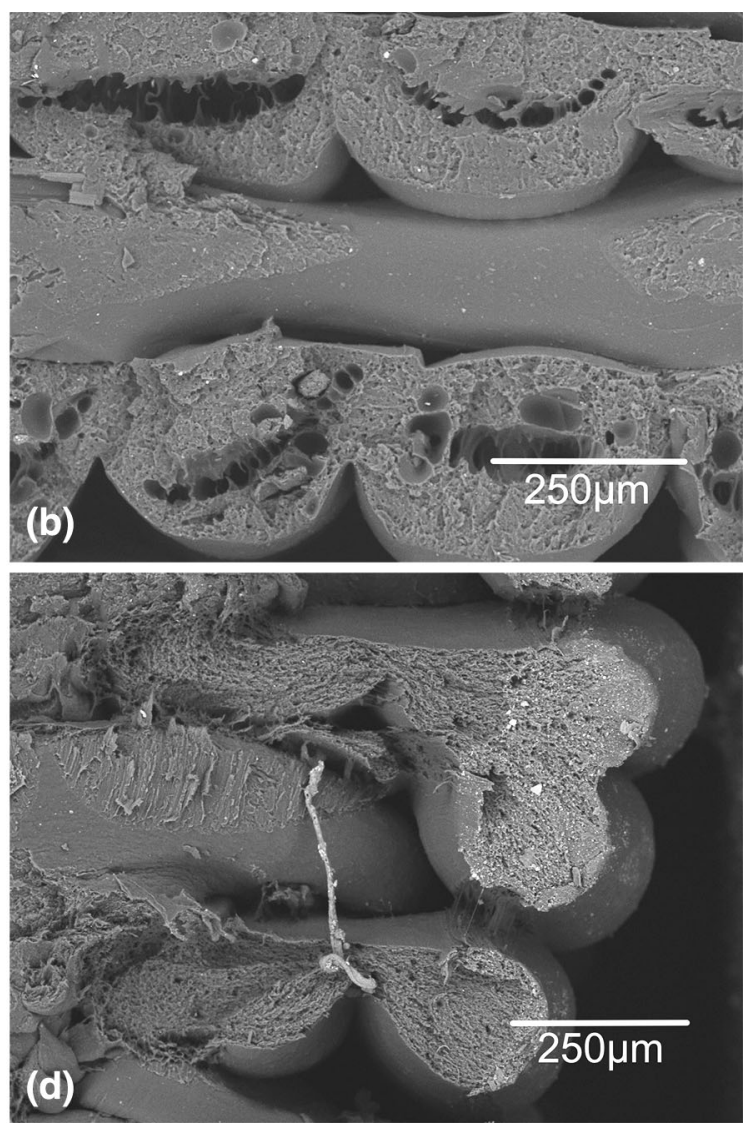

Fig. 9 SEM images of the fractures of the XYZ samples: low magnification. (a) ABS, (b) ABS and jute fiber, (c) $\mathrm{ABS}$ and TiO ${ }_{2}$, and (d) $\mathrm{ABS}$ and TPE 

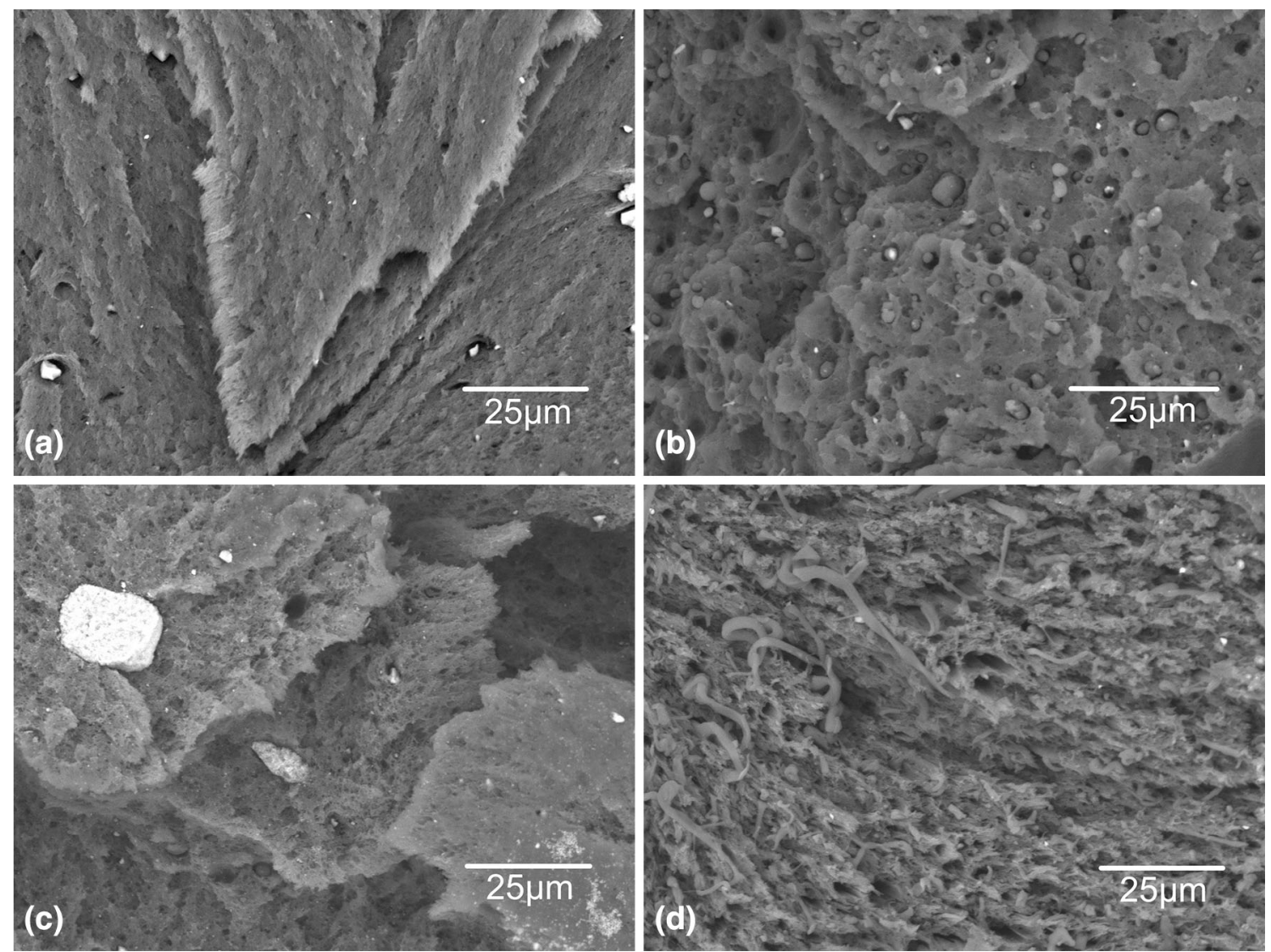

Fig. 10 SEM images of the fractures of the XYZ samples: high magnification. (a) ABS, (b) ABS and jute fiber, (c) $\mathrm{ABS}_{\text {and }} \mathrm{TiO} \mathrm{O}_{2}$, and (d) $\mathrm{ABS}$ and TPE

through the twin-screw extrusion process, the process of material extrusion 3DP entails subjecting the monofilament to an extrusion process at temperatures above the decomposition temperature of jute. It can be observed in Fig. 10b that little remains of the original morphology of the fibers, most likely due to the fact that mixing process inside the extruder exposes the fibers to shear forces that break down the fibers, and because of the decomposition process already mentioned. The voids inside the material provide an explanation for the decrease in UTS. The increase in strain observed in the plastic deformation region of the stress-strain curve plot may be due to the particles having a higher freedom to easily reallocate themselves inside the matrix [16] leading to an ability to sustain more plastic deformation than ABS alone.

The presence of $\mathrm{TiO}_{2}$ reduces the freedom of the plastic macromolecules to slide over one another, producing a fracture surface indicative of a brittle failure. When micrographs of the $\mathrm{TiO}_{2}$ composites are compared with the other material types as observed in Figs. 9c and 10c, it is notable that the fracture surface is nearly flat and without regions of deformation. No deformed fibrils are present. Abrupt steps and striations are observed as indicated with white arrows. The observed results are congruent to the reduction of the plastic region observed on the stress-strain curve plot for samples fabricated from the $\mathrm{ABS} / \mathrm{TiO}_{2}$ material system. Agglomerated $\mathrm{TiO}_{2}$ powder with diameters up to $25 \mu \mathrm{m}$ can be seen in the micrographs indicated with black arrows, though initial characterization of the powder revealed the nominal particle diameter to be an order of magnitude smaller, on the order of $50 \mathrm{~nm}$, indicating a problem with particle dispersion during the compound of the composite monofilament. The use of silanes has proven to be a successful avenue in improving the dispersion and adhesion of $\mathrm{TiO}_{2}$ particles within a polymer matrix [18]. As mentioned before, the $\mathrm{ABS} / \mathrm{TiO}_{2}$ system exhibited the highest value for the Young's modulus. The variation of the Young's Modulus for the other materials tested is negligible compared with ABS alone.

The ABS/TPE blend exhibited characteristics indicative of ductile fracture after undergoing the highest amount of plastic deformation of the four compositions (Figs. 9d, 10d). Figure $10 \mathrm{~d}$ shows one of the craze regions with multiple torn-off fibrils with diameters between 1 and $5 \mu \mathrm{m}$ coinciding with a normal stress zone, typical of elastomeric materials [16]. 

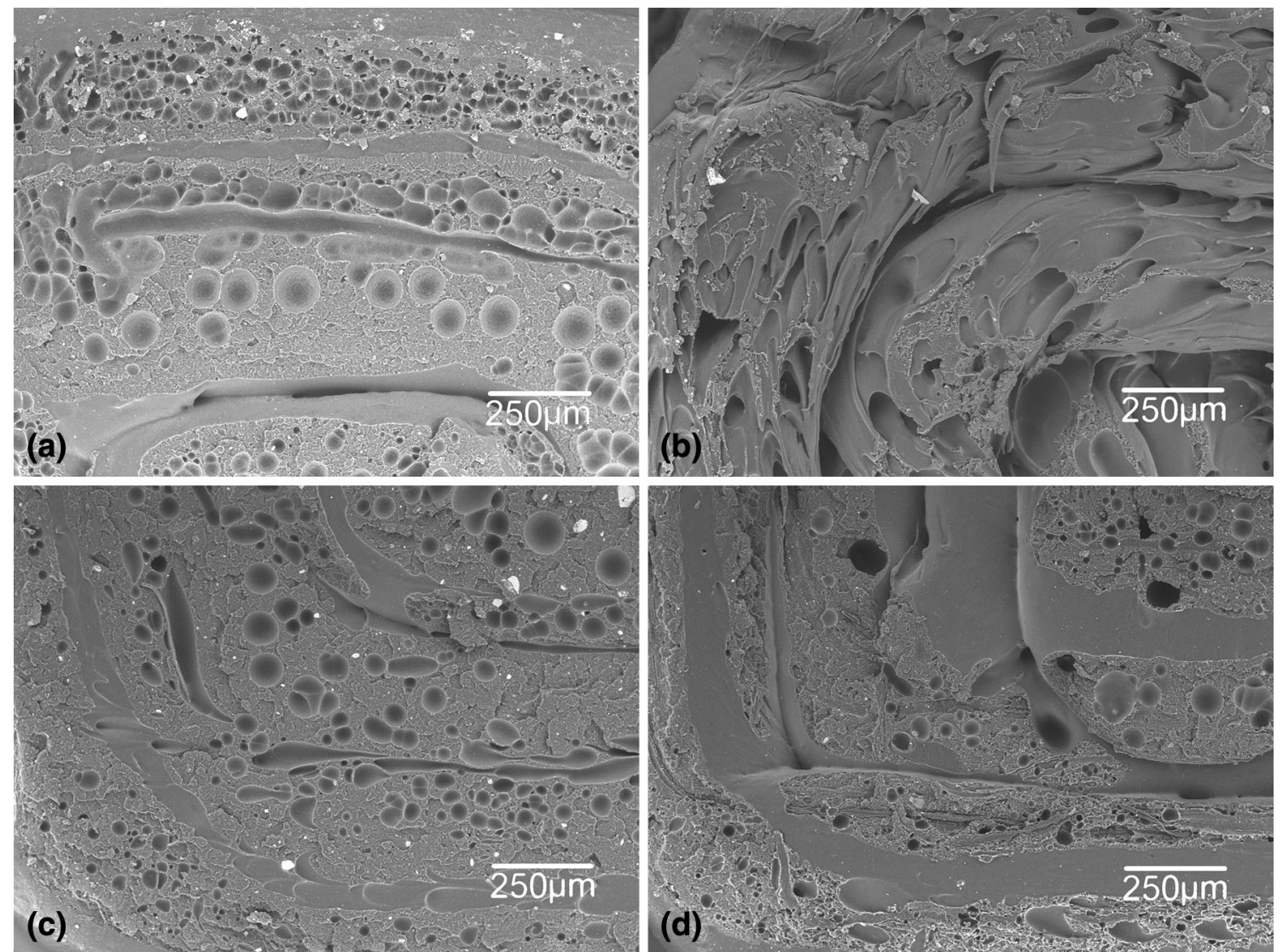

Fig. 11 SEM images of the fractures of the ZXY samples: low magnification. (a) ABS, (b) ABS and jute fiber, (c) $\mathrm{ABS}$ and $\mathrm{TiO}{ }_{2}$, and (d) $\mathrm{ABS}$ and TPE

\section{Fractography of Components Fabricated in the ZXY Direction}

The fracture surfaces observed on tensile specimens fabricated from the four material types that were printed in the ZXY direction (Figs. 11, 12) present different characteristics compared to those fabricated in the XYZ direction. The fracture surfaces in the case of the ZXY direction-built specimens exhibit brittle characteristics and large cavities with the exception of the specimens loaded with jute fiber.

The fracture surfaces of the sample printed from ABS are shown in Figs. 11a and 12a and exhibit brittle fracture surface characteristics in contrast to the ductile fracture characteristics observed on the fracture surface of XYZ direction. The formation of circular cavities with diameters from $5 \mu \mathrm{m}$ in the outer section of the fracture to larger diameters of up to $50 \mu \mathrm{m}$ on the inside of the fracture can be observed. Another notable feature of the fracture surface is the lack of the presence of fibrils (12a). A key characteristic of this fracture surface is a flake-like morphology indicative of an extended normal stress zone. The fracture surface of $\mathrm{ABS} / \mathrm{TiO}_{2}$ composite possesses similar characteristics to that of the ABS specimen (Figs. 11c, 12c). However, the
$\mathrm{ABS} / \mathrm{TiO}_{2}$ fracture surface exhibited a lower number of cavities with a more uniform diameter distribution along the surface. The fracture surface of the sample fabricated from the ABS/TPE blend showed similar brittle fracture characteristics to $\mathrm{ABS}$ and the $\mathrm{ABS} / \mathrm{TiO}_{2}$ composite (Figs. 11d, $12 \mathrm{~d}$ ), but with fewer voids of smaller diameter on average. The increase in tensile strength and modulus at the expense of ductility is a common tradeoff in the fabrication of composite materials. Moreover, the presence of $\mathrm{TiO}_{2}$ particles acts as a stress concentrator. The particles act as barriers to the propagation of microfractures produced in the matrix during the plastic deformation of the sample. As the cracks are not able to progress when they reach a particle, the effective ductility is reduced. The accumulation of microfractures within a certain region will eventually lead to a brittle macrofracture when the rupture occurs.

The fracture surface of the ABS/jute fiber composite was the only material type to exhibit ductile-like fracture characteristics (Figs. 11b, 12b) among the sample types printed in the ZXY direction. The ductile-like morphology of the fracture surface is misleading as it was most likely caused by the transverse rupture of the printed filament (Fig. 13). The voids observed in the XYZ direction-built 

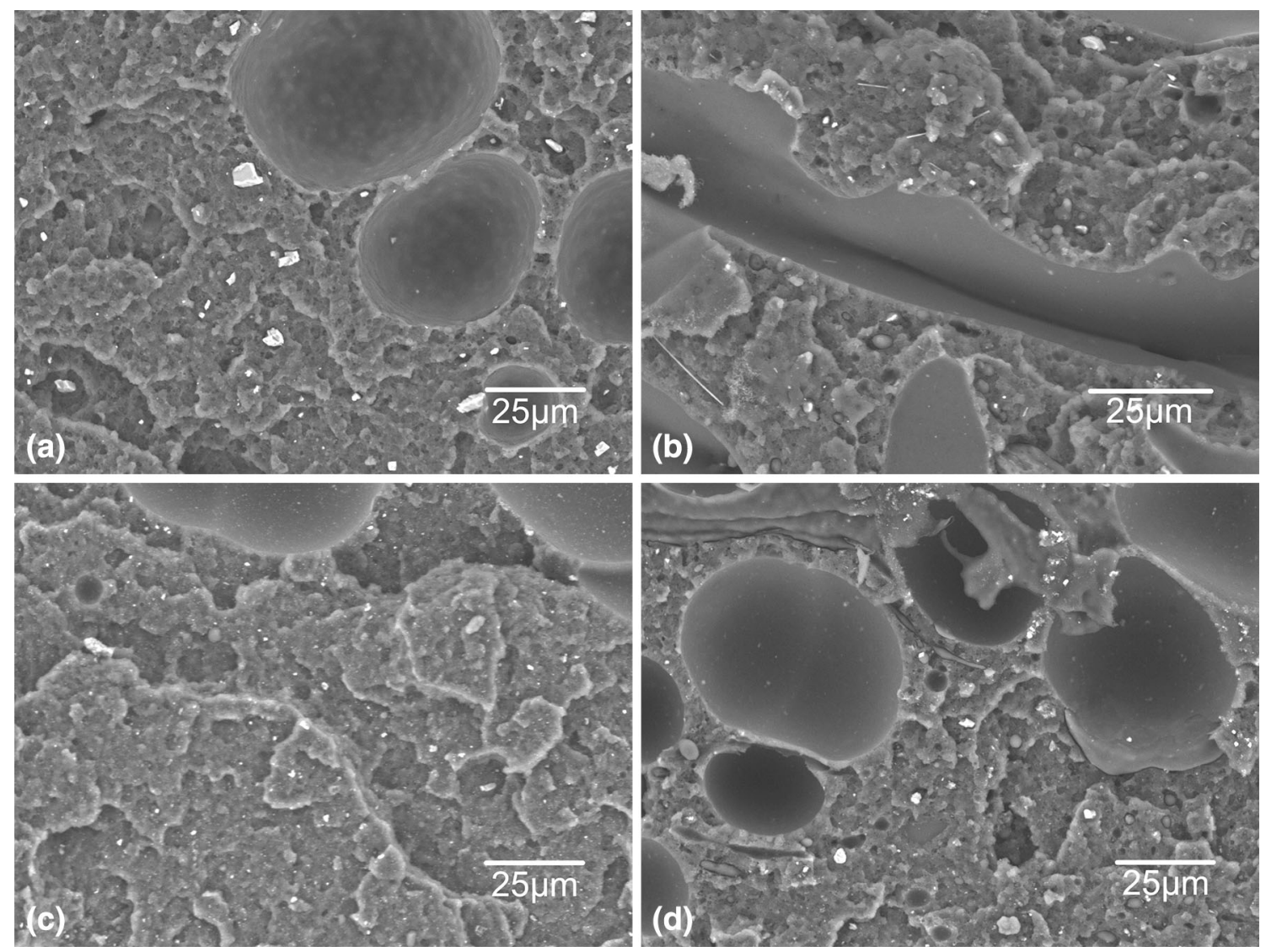

Fig. 12 SEM images of the fractures of the ZXY samples: high magnification. (a) ABS, (b) ABS and jute fiber, (c) $\mathrm{ABS}$ and $\mathrm{TiO} \mathrm{O}_{2}$, and (d) $\mathrm{ABS}$ and TPE

specimen were oriented perpendicular to the applied stress for samples printed in the ZXY direction. Though the morphology of the fracture surface resembles and therefore, suggests a torsional fracture, the deformation was produced during the fabrication of the test specimens and follows the direction of print deposition. The present vacancies were caused by the decomposition jute as discussed previously. During the fracture process, the voids deformed further, and crazes were generated from these voids. The normal stress region is reduced with respect the other materials tested, but still no fibrils were observed indicating the fracture was brittle in nature. The addition of jute fiber provokes the lowest Young's modulus of all the materials, which correlates with the higher deformation. For samples fabricated in the ZXY direction, there are two failure modes present: (1) the failure of the inter-layer bond between printed rasters as observed in the $\mathrm{ABS}, \mathrm{ABS} / \mathrm{TiO}_{2}$, and ABS/TPE blend; and (2) transfilament rupture caused by the voids present in the ABS/Jute fiber composite.

The data plots represented in Fig. 14 summarize the results for the UTS, modulus, and elongation to break for all the material systems tested for samples manufactured in both the vertical and horizontal printing directions. Figure 14a

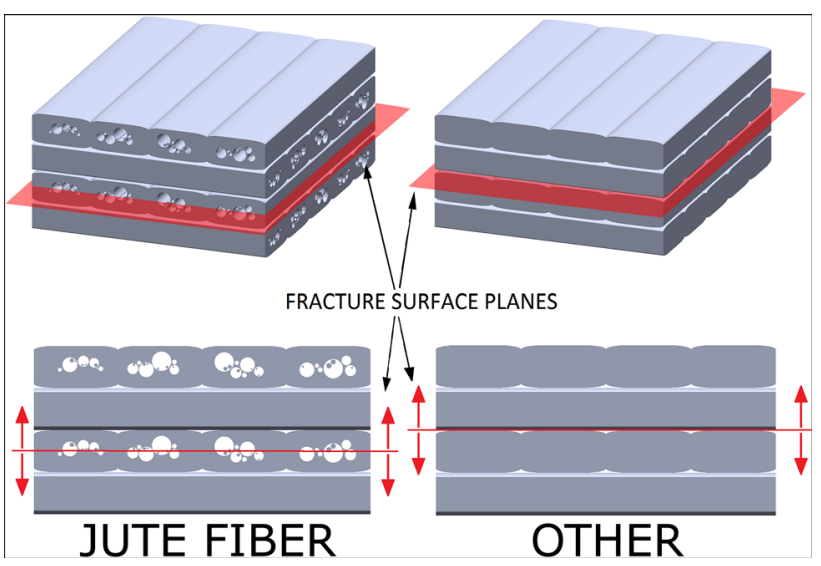

Fig. 13 Schematic of fracture plains for jute fiber compared with other composites

shows that only the addition of $\mathrm{TiO}_{2}$ particles leads to an improvement in UTS compared with the other experiments; however, of notable interest is the reduction in the difference between vertical and horizontal UTS values for the ABS/ TPE blend, indicating a decrease in mechanical property anisotropy for components printed from this material system. The addition of $\mathrm{TiO}_{2}$ had the effect of increasing the 

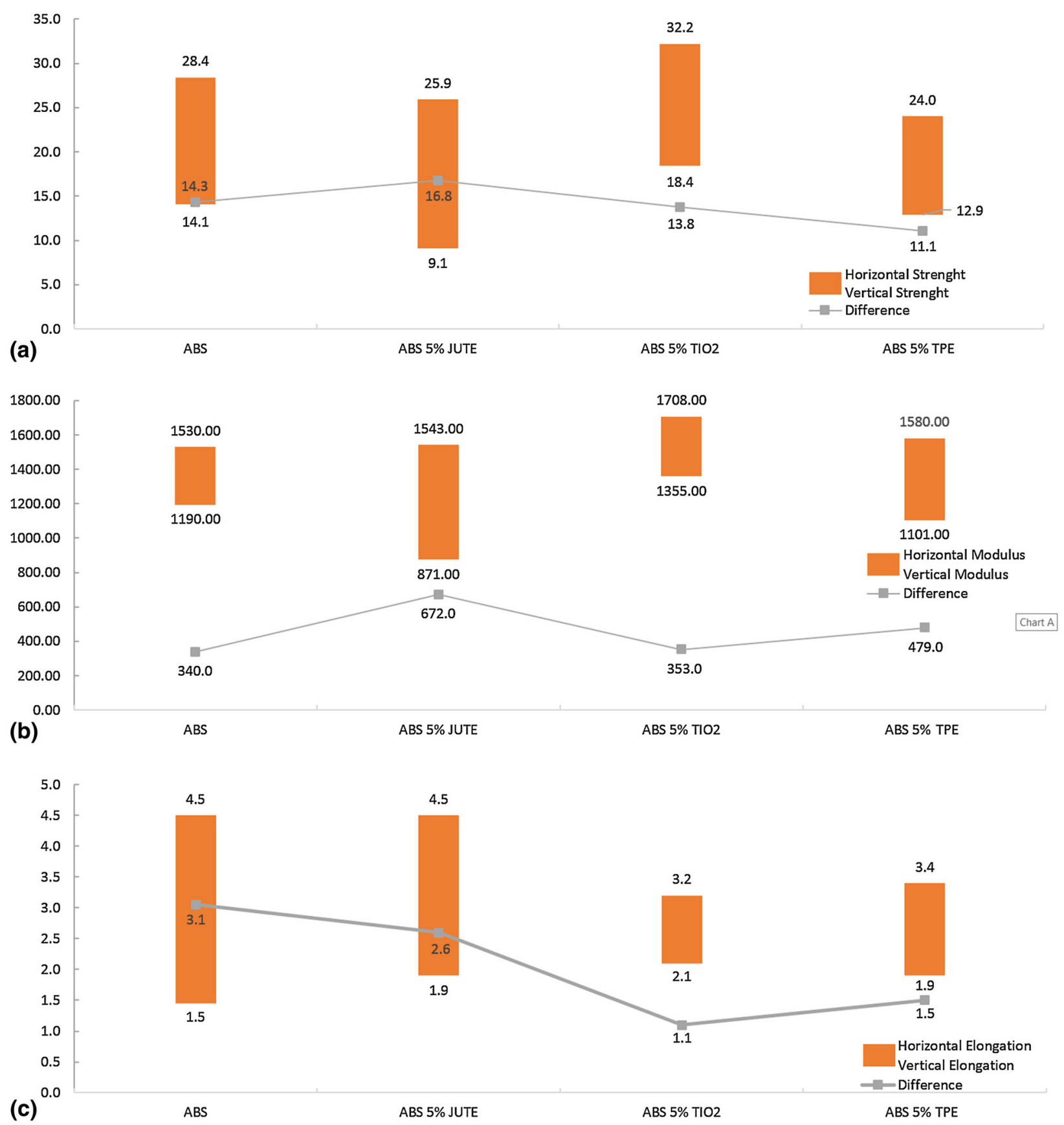

Fig. 14 Differences in the results of XYZ and ZXY samples for: (a) UTS, (b) modulus, and (c) elongation to break

modulus as can be seen in Fig. 14b. Figure 14c also indicates reduction in the difference of the elongation to break between the vertical and the horizontal samples for both the materials with the addition of $\mathrm{TiO}_{2}$ and TPE.

\section{Conclusions}

The addition of reinforcing agents to ABS PMC has an effect on the mechanical properties and fracture surface characteristics of tensile specimens compared with pure ABS. Instead of the expected ductile fracture behavior, the addition of additives led to fracture surfaces that exhibited brittle characteristics. These systems were ABS with 5 wt. $\%$ jute fiber, $\mathrm{ABS}$ with 5 wt. $\% \mathrm{TiO}_{2}$, and $\mathrm{ABS}$ with 5 wt. $\%$ TPE. The $\mathrm{ABS} / \mathrm{TiO}_{2}$ system displayed higher UTS compared with pure ABS and the other composite systems studied in this paper, but the fracture characteristics of this material system indicated brittle fracture. The present study gives an indication of the manipulation of mechanical 
failure characteristics of components fabricated from 3Dprintable composites and polymeric blends.

The fracture surfaces for parts fabricated in the two build orientations exhibited different morphological characteristics, most notably, the presence of cavities, which was more abundant for parts built in the ZXY direction, most likely due to a failure of the interface between printed layers. It is known that the mechanical strength for parts fabricated in the ZXY direction is lower as opposed to parts fabricated in the $\mathrm{XYZ}$ direction $[14,15]$. Here, the same behavior was observed; however, the results of the ABS/TPE system suggest a pathway toward reducing the characteristic anisotropy of components fabricated form material extrusion 3DP.

Acknowledgments This research was performed at The University of Texas at El Paso (UTEP) within the W.M. Keck Center for 3D Innovation (Keck Center), expanding recently to over 13,000 sq. ft. as a result of funding from the State of Texas Emerging Technology Fund and providing access to state-of-the-art facilities and equipment. Specially appreciated is the help of Nubia Zuverza and Carmen Rocha, always available whenever needed.

\section{References}

1. B. Berman, 3-D printing: the new industrial revolution. Business Horiz. 55(2), 155-162 (2012)

2. J.L. Chulilla-Cano, The cambrian explosion of popular 3D printing. IJIMAI 1(4), 30-32 (2011)

3. S. Masood, W. Song, Development of new metal/polymer materials for rapid tooling using fused deposition modeling. Mater. Des. 25(7), 587-594 (2004)

4. M. Nikzad, S.H. Masood, I. Sbarski, Thermo-mechanical properties of a highly filled polymeric composites for fused deposition modeling. Mater. Des. 32(6), 3448-3456 (2011)

5. A. Safari, E.K. Akdogan, Rapid prototyping of novel piezoelectric composites. Ferroelectrics 331(1), 153-179 (2006)
6. M.L. Shofner, K. Lozano, F.J. Rodríguez-Macías, E.V. Barrera, Nanofiber-reinforced polymers prepared by fused deposition modeling. J. Appl. Polym. Sci. 89(11), 3081-3090 (2003)

7. M.L. Shofner, F.J. Rodríguez-Macías, R. Vaidyanathan, E.V. Barrera, Single wall nanotube and vapor grown carbon fiber reinforced polymers processed by extrusion freeform fabrication. Composites 34(12), 1207-1217 (2003)

8. S.-Y. Fu, X.-Q. Feng, B. Lauke, Y.-W. Mai, Effects of particle size, particle/matrix interface adhesion and particle loading on mechanical properties effects of particle size, particle/matrix interface adhesion and particle loading on mechanical properties of particulate-polymer composites. Composites 39, 933-961 (2008)

9. S. Tjong, Structural and mechanical properties of polymer nanocomposites. Mater. Sci. Eng. 53, 73-197 (2006)

10. T. Huq, A. Khan, T. Akter, N. Noor, K. Dey, B. Sarker, M. Saha, R.A. Khan, Thermo-mechanical, degradation, and interfacial properties of jute fiber-reinforced PET-based composite. J. Thermoplast. Compos. Mater. 24(6), 889-898 (2011)

11. S. Wu, Phase structure and adhesion in polymer blends: a criterion for rubber toughening. Polymer 26(12), 1855-1863 (1985)

12. ASTM Standard D6110, Standard Test Method for Tensile Properties of Plastics, (ASTM International, West Conshohocken, 2010)

13. S.-H. Ahn, M. Montero, D. Odell, S. Roundy, P.K. Wright, Anisotropic material properties of fused deposition modeling abs. Rapid Prototyping 8(4), 248-257 (2002)

14. A. Bellini, S. Güçeri, Mechanical characterization of parts fabricated using fused deposition modeling. Rapid Prototyping J. 9(4), 252-264 (2003)

15. O.S. Es-Saida, J. Foyosa, R. Noorania, M. Mendelsona, Effect of layer orientation on mechanical properties of rapid prototyped samples. Mater. Manuf. Process. 15(1), 107-122 (2000)

16. L. Engel, H. Klingele, G.W. Ehrenstein, H. Schaper, An Atlas of Polymer Damage: Surface Examination by Scanning Electron Microscope (Prentice-Hall, Inc., Englewood Cliffs, 1981)

17. J. Gassan, A.K. Bledzki, Thermal degradation of flax and jute fibers. J. Appl. Polym. Sci. 82(6), 1417-1422 (2001)

18. M. Altan, H. Yildirim, A. Uysal, Tensile properties of polypropylene/metal oxide nano composites. Online J. Sci. Technol. 1(1), 25-29 (2011) 